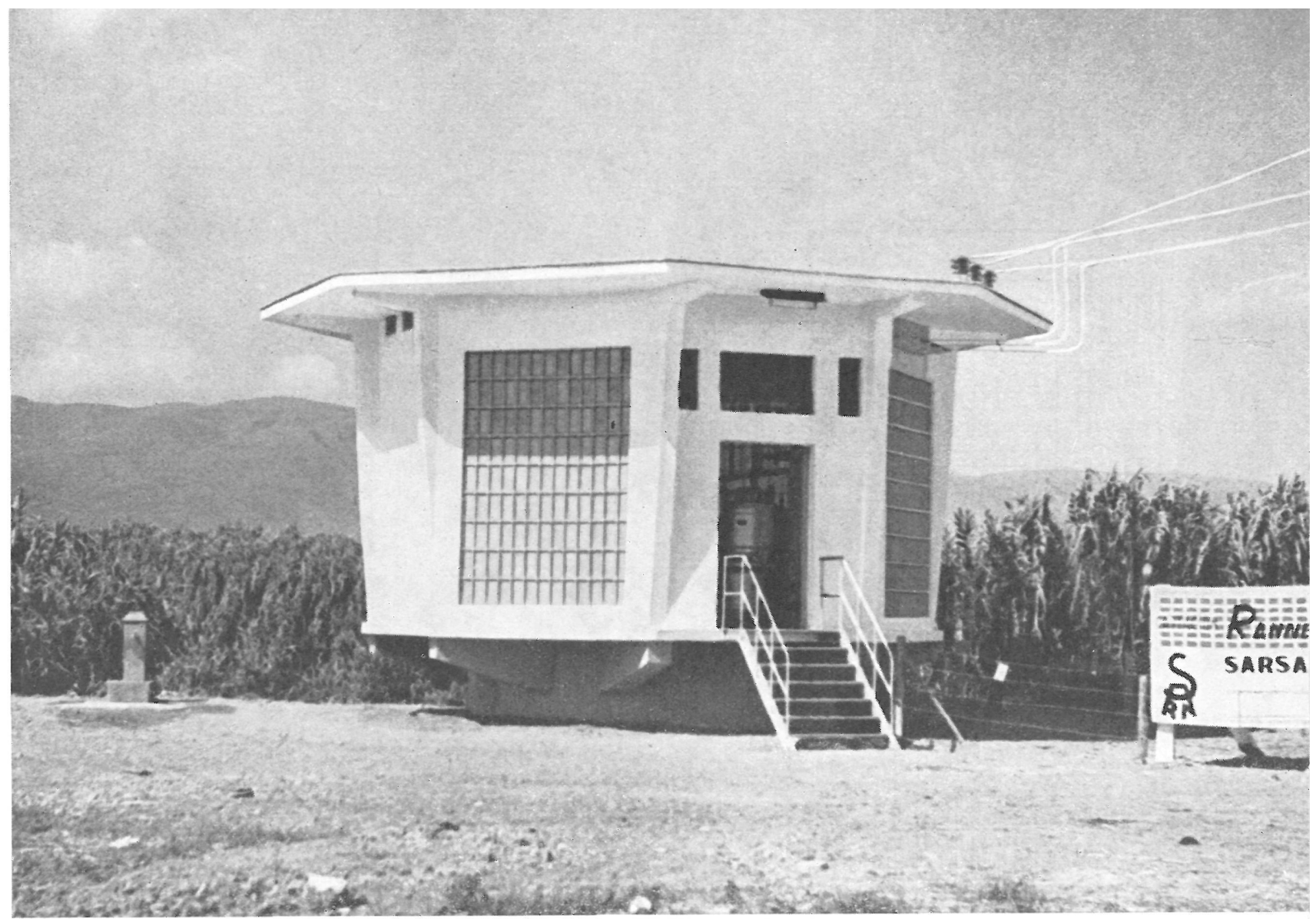

$534 \cdot 1$

\title{
captación por el sistema Ranney para aumentar el abastecimiento de agua de Málaga
}

Para aliviar el problema de falta de agua, cada vez más agudizado, el Excmo. Ayuntamiento de Málaga, apoyado por las altas Autoridades y en estrecha colaboración con la Dirección de Obras Hidráulicas y la Confederacion del Rio Guadalhorce, previo informe del competente Ingeniero de Caminos D. Alejandro Miró Nadal, decidió la ejecución de una captación subálvea en una de las márgenes del río Guadalhorce, en las proximidades del sifón de la conducción de Torremolinos a Málaga.

El sistema de captación es el de pozo colector con drenes horizontales, construído según la patente americana Ranney Method International, Inc., $y$ es el primero de esta clase que funciona en España.

La obra se ha realizado en un tiempo récord, ya que, desde la orden de comienzo hasta su puesta en marcha, solamente han transcurrido cuatro meses.

El nuevo abastecimiento, que complementa el antiguo de Torremolinos, está en funcionamiento desde el 1 de julio.

Construcción de la sala de máquinas.

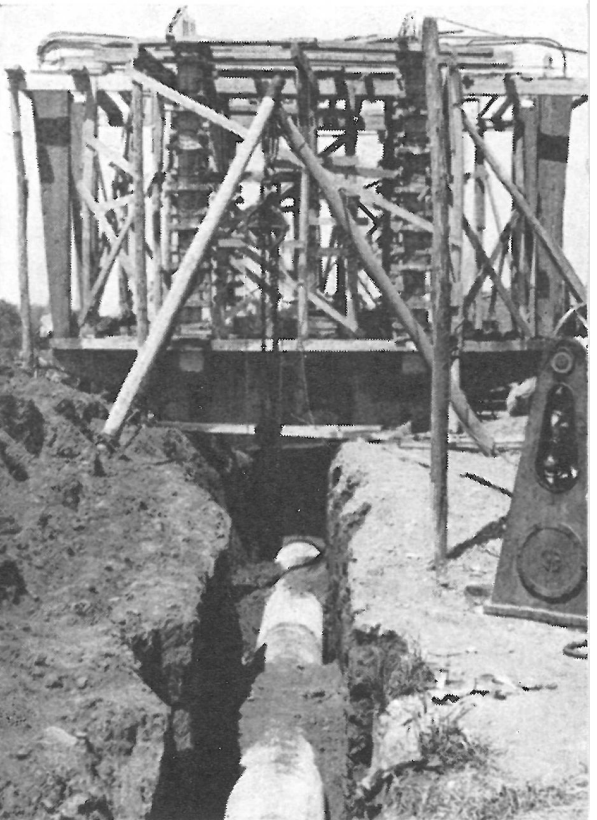




\section{Pozo colector}

El pozo colector es un cajón cilíndrico de hormigón fuertemente armado, de $4 \mathrm{~m}$ de diámetro, hincado en el terreno por el procedimiento indio hasta la capa impermeable, situada a una profundidad de 9,20 metros.

En el primer anillo del cajón se dejaron preparadas las lumbreras necesarias para la hinca posterior de los drenes radiales horizontales.

$\mathrm{Su}$ fondo está sellado por una solera de hormigón sumergido, de un espesor en el centro de 1,50 metros.

Queda, pues, el pozo colector constituído por un vaso herméticamente cerrado, impermeable, hincado en la capa de acarreos, al que tiene acceso el agua infiltrada por los drenes horizontales, que se hincan en el terreno a través de las lumbreras situadas en el primer anillo.

\section{Drenes horizontales}

A través de las lumbreras se han hincado nueve drenes horizontales.

Los drenes son tubos de chapa de acero, de $216 \mathrm{~mm}$ de diámetro, ranurados. Su penetración en el terreno se ha realizado por medio de dos gatos hidráulicos gemelos, de una potencia de 100 toneladas cada uno, que se apoyan en el muro del pozo.

Estos drenes se han hincado por tramos. El extremo del primer tramo de cada dren va provisto de una cabeza de avance especial. Con el fin de impedir la penetración del agua en el interior del pozo, se dispone, entre el dren y la boca de salida, un pelración tubería de extracción de arenas.

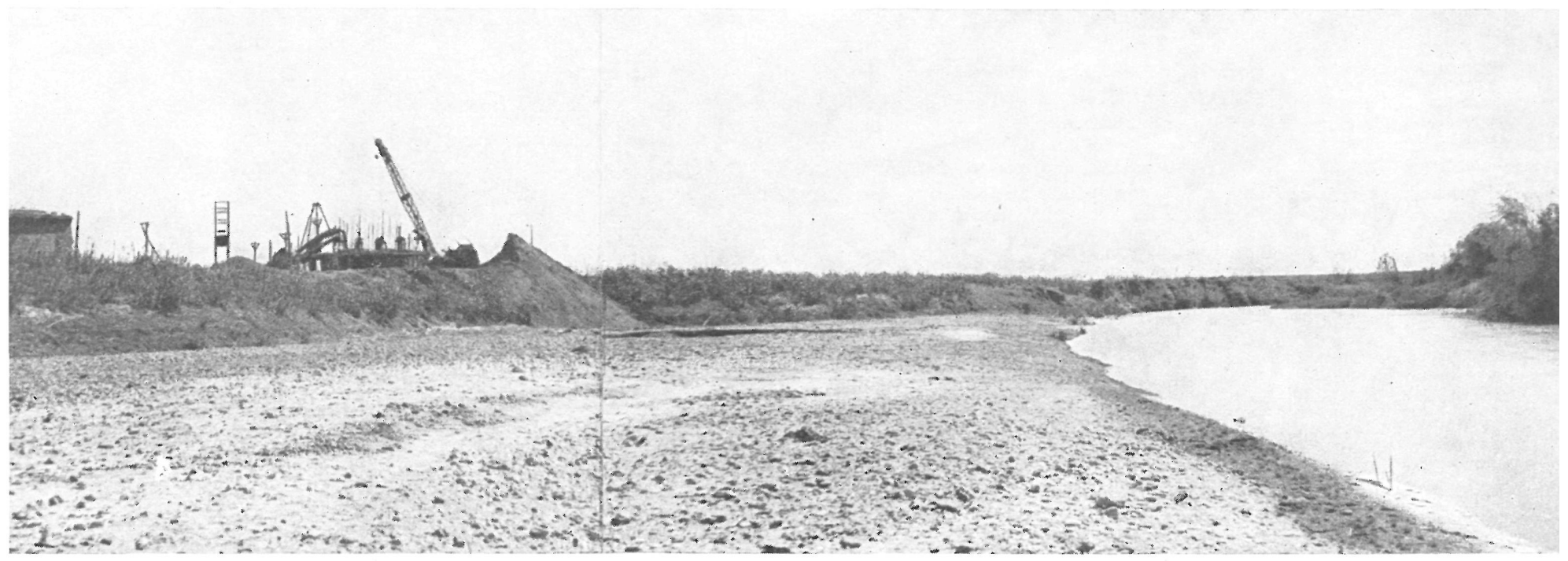

Iniciación de la obra.

El agua de la capa freática, al penetrar por la cabeza de avance, llega al pozo atravesando la tubería de desarenado. Como consecuencia de la presión estática, la velocidad del agua en este punto aumenta, arrastrando todos los finos que se encuentran en la proximidad de la punta, haciendo posible la hinca.

Este agua, que contiene arenas en gran cantidad, se ha extraído del pozo por medio de una bomba después de haber dejado sedimentar las arenas. Durante la hinca se han tenido instaladas tres bombas.

Por efecto del desarenado, durante y después de la hinca, se ha llegado a crear, alrededor del tubo ranurado, un recubrimiento de gravas de un diámetro del orden de 1,5 a $2 \mathrm{~m}$, cuyo efecto es aumentar notablemente la afluencia del agua hacia el dren.

Una vez hincado cada dren a la longitud prevista, se ha dispuesto en su embocadura una válvula de compuerta, que puede accionarse desde una plataforma auxiliar y que permite cerrarle herméticamente.

Los drenes se han hincado hasta longitudes de 51 metros. En total, $315 \mathrm{~m}$ de tubería ranurada. El objeto de disponer de tal longitud total de drenes es el de asegurarse de que, bombeando por el pozo cantidades del orden de 300 litros por segundo, no se produzcan arrastres de arena ni colmataciones en los mismos. 
construcción

\section{Plataforma auxiliar de mandos}

Al nivel de $6 \mathrm{~m}$ sobre el plano horizontal de drenes se ha dispuesto, en el interior del pozo, una plataforma de chapa estriada soportada por viguetas empotradas en el revestimiento, desde la cual se pueden accionar, por medio de volantes que transmiten su movimiento por intermedio de varillaje tubular, las válvulas de compuerta de que va provisto cada dren. Estas válvulas los drenes que se desee.

También va situado al nivel de esta plataforma la salida de las bombas, con sus correspondientes válvulas de compuerta y de retención. Las salidas de estas bombas se han unido por una pieza especial de chapa soldada-pantalón-a la tubería de impulsión de hormigón armado vibrocentrifugado con chapa intermedia, que une el pozo con la conduccion general de Torremolinos a Málaga. Los mandos de las válvulas de compuerta de las bombas se han prolongado por medio de varillajes tumáares hasta el piso de la sala de máquinas

\section{Superestructura}

El tubo que forma el revestimiento del pozo colector se ha prolongado 1,50 metros sobre la superficie del terreno para aislar su interior y la sala de máquinas de posibles avenidas.

Sobre la extremidad superior de este tubo, y soportada por cuatro vigas, cuyos extremos vuelan $1,90 \mathrm{~m}$ de la circunferencia exterior del mismo, se ha construido una superestructura para alojamiento de la maquinaria. Esta superestructura togonal.

La parte central del piso de la caseta, y limitada por las vigas soporte, se ha dejado diáfana y solamente cubierta por placas de chapa estriada meté en perfiles. Tanto este piso pueden pueden

El techo de esta superestructura está soportado por cuatro pórticos cruzados que se apoyan sobre las ménsulas de las vigas soporte del piso y es capaz de soportar pesos concentrados de hasta una portar pesos concentrados de hasta montaje de las máquinas. IO desintermedios, entre los ocho pilares de estos pórticos, se han construído de hormigón traslúcido-pavés de vidrioy de ladrillo enfoscado y pintado.

\section{Sala de máquinas}

La caseta octogonal que constituye la superestructura de la obra está dedicada a sala de máquinas.

En su interior va dividida en tres partes, separadas por dos mamparas de partes, separadas por dos mamparas de piso hasta , que se extienden desde el l aparellaje eléctrico, de alta tension del pasillo central.
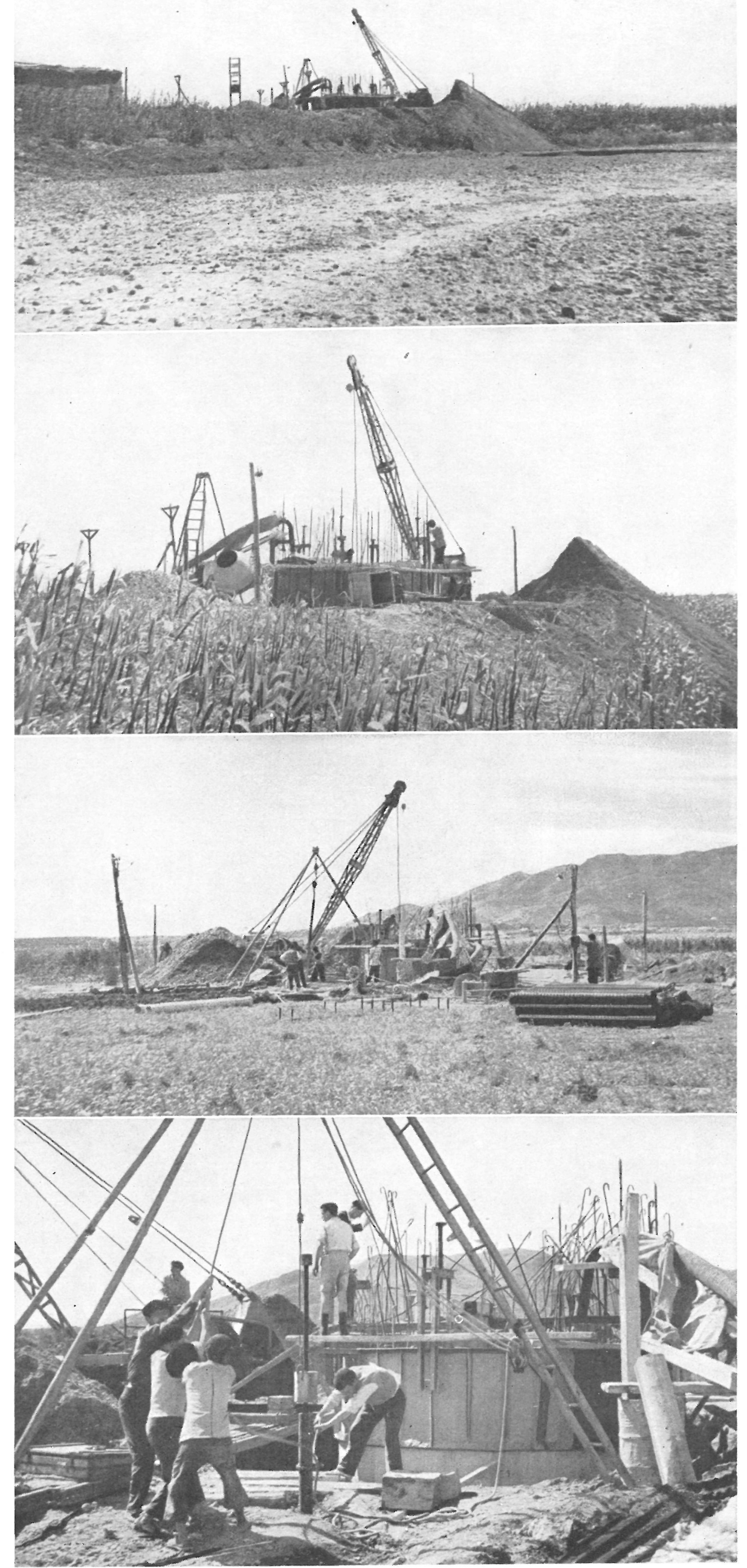


\section{sección y planta}

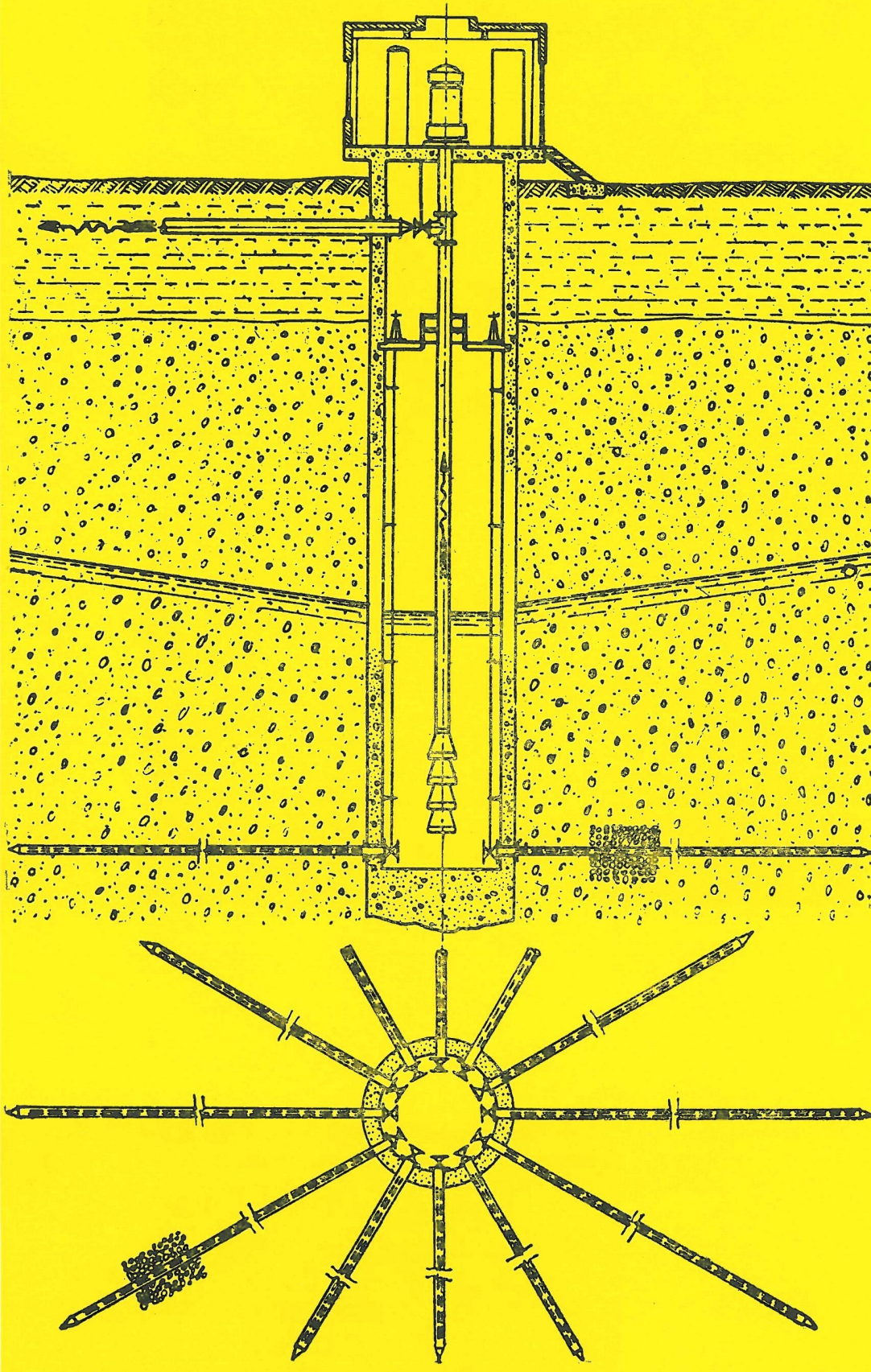

Apoyados en la estructura metálica central van dos grupos electrobombas Worthington, tipo 12 QH, de seis fases, con motores de $125 \mathrm{CV}$, para elevar 100 litros por segundo a una altura manométrica de 66 metros. Situados en la pared del pasillo se hallan dos armarios metálicos con los cuadros de mando a distancia y señalización luminosa y guarda motores para la protección de la maniobra eléctrica de las bombas.

En las dos celdas, a ambos lados del pasillo central, va todo el aparellaje eléctrico de alta tensión y dos transformadores 125 KVA cada uno, a $33.000 / 380 \mathrm{~V}$

\section{Pruebas de la instalación}

El día 30 de junio se pusieron en marcha los grupos electrobombas en régimen de pruebas, manteniendo abiertos los desagües de la conducción general para limpiar las tuberías.

A partir del 1 de julio se cerraron los desagües y se empezó a enviar agua a los depósitos de Málaga.

Durante las pruebas se trabajó con una y dos bombas alternativamente y durante algunos momentos con una tercera bomba auxiliar de las empleadas durante las obras, llegando a extraer por encima de 350 litros por segundo y produciendo el descenso máximo admisible en el pozo.

En el transcurso de estas pruebas tan extremadas se tomaron muestras de aguas a los distintos regímenes de bombeo, resultando absolutamente claras. exentas por completo de turbiedad, quedando con ello demostrada la ausencia de arrastres de finos. 


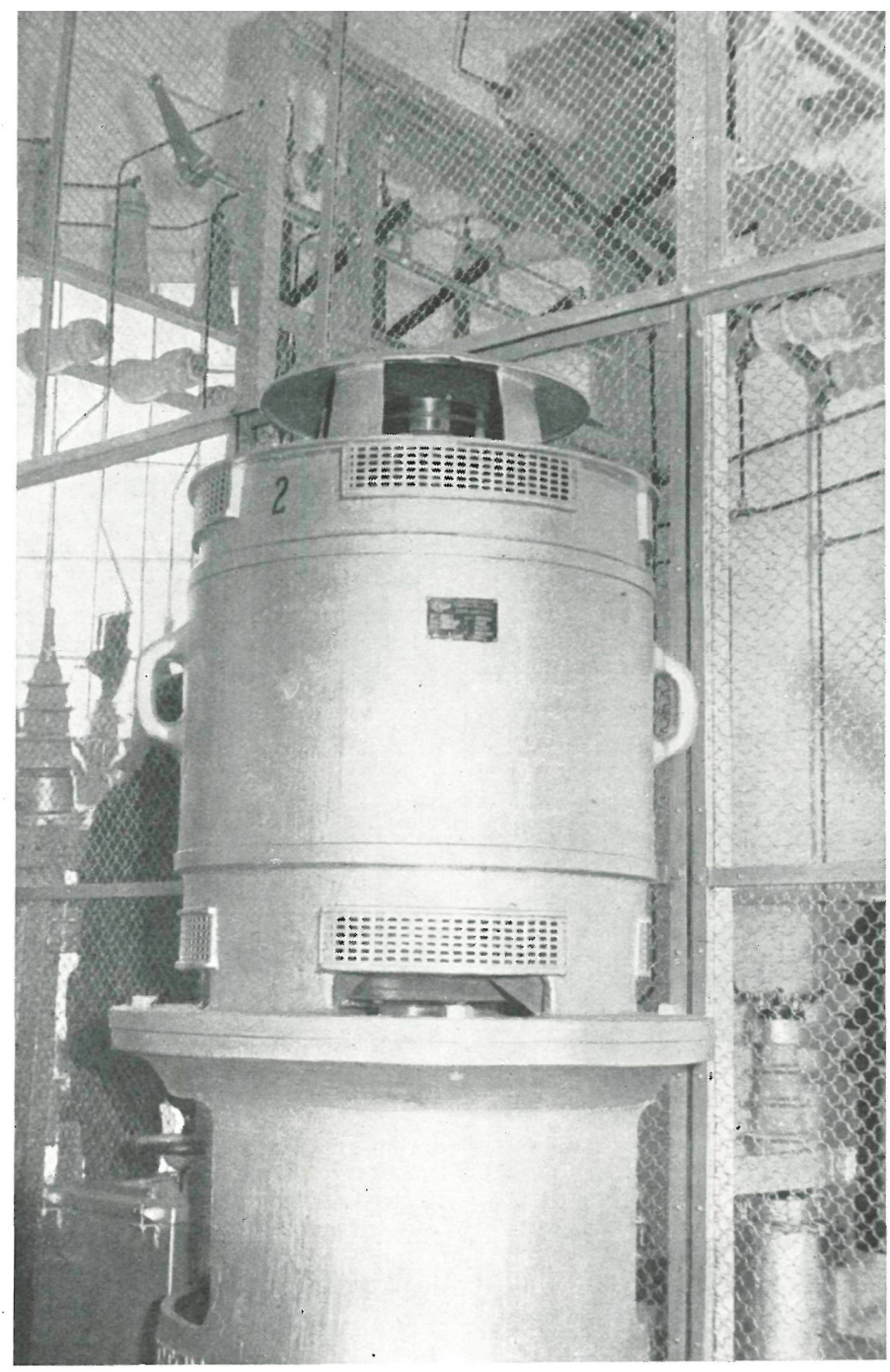

Por lo que se refiere a colmataciones e incrustaciones, el tiempo ha de demostrarnos, al igual que en otras instalaciones similares extranjeras, que, debido a la tan lenta velocidad de infiltracion en los drenes, para los caudales incrustaciones, ya que, en esas velocidades tan lentas, no son de temer ni arrastres ni desprendimientos de gas carbónico debido a caídas bruscas de presión que gas lugar a la precipitación de los bicarbonatos en que dan carbonatos, con la consiguiente obstrucción de las ranuras de los drenes.

Se mantuvo alternativamente el trabajo de una y dos bombas durante los días $1,2,3,4$ y 5 , y a partir de éste se ha seguido bombeando con una sola bomba, habiéndose por segundo.

En el período de tiempo que la instalación lleva funcionando ha quedado demostrado que, a pesar de la pequeña altura hidrostática sobre el nivel de los drenes, inferior a $3 \mathrm{~m}$, de la naturaleza desfavorable de los acarreos, que presentan intercalaciones horizontales de zonas fangosas y arcillosas, y de la época del año, continuación del anterior extremadamente seco, el caudal que se obtiene con regularidad por este pozo Ranney es enormemente mayor que el que pudiera obtenerse con cualquier otro sistema de captación subálvea.

\section{Resumen}

El sistema adoptado para paliar el problema de escasez de agua en Málaga, que, especialmente para este verano, presentaba caracteres muy alarmantes, ha venido a auun tercio y ha resultado ser la solución más rápida, eficaz y económica.

En nuestra opinión, otra unidad de este tipo, convenientemente localizada-más aguas arriba en las márgenes del río Guadalhorce-, después de un detallado estudio de reconocimiento y aforos, daría a Málaga una tranquilidad completa.

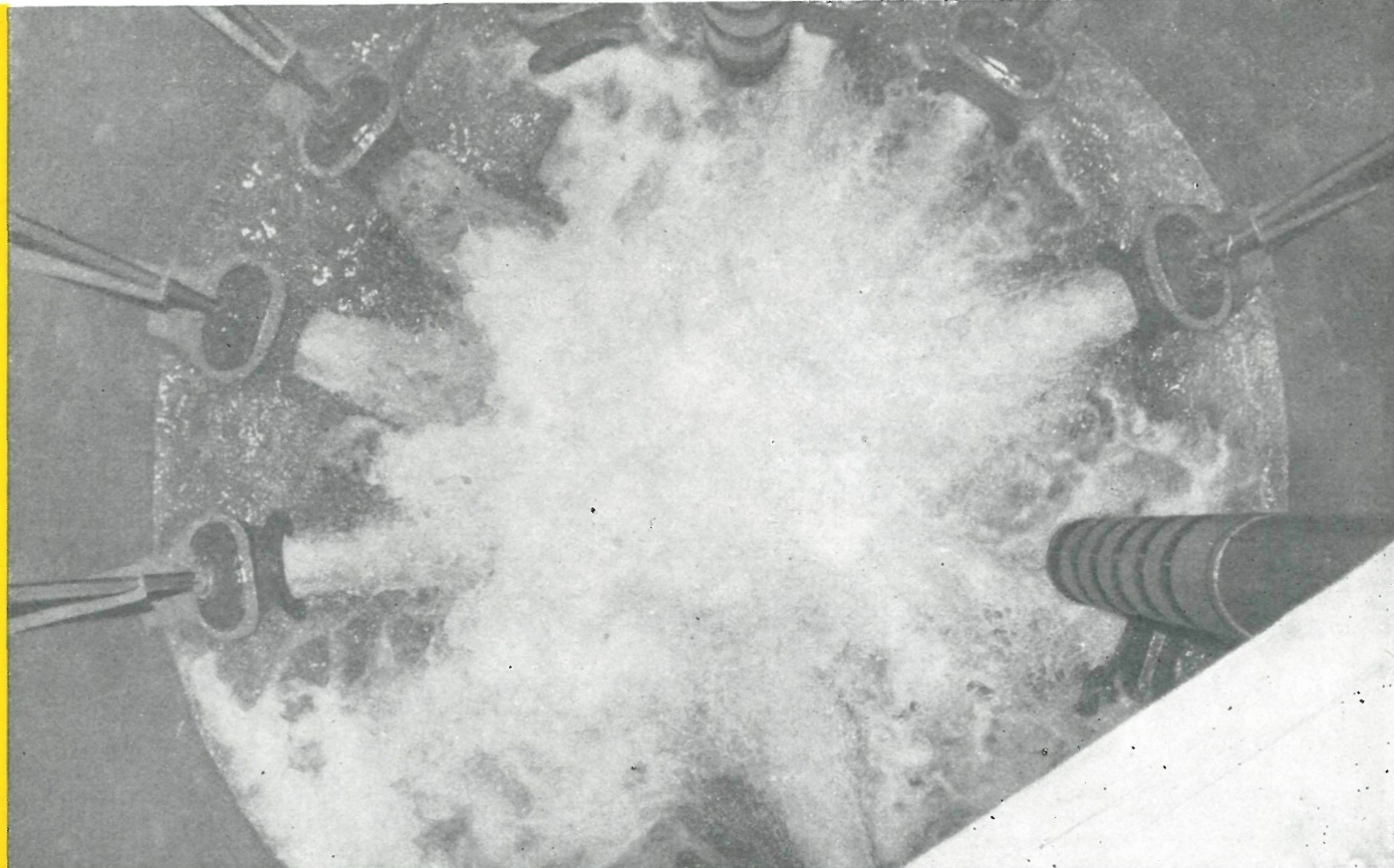

